Génét. Sél. Evol., 1984, 16 (2), 239-244.

\title{
Note
}

\section{Un cas probable de recombinaison dans le système de groupes sanguins $\mathbf{S}$ des bovins}

\author{
F. GROSCLAUDE \\ I.N.R.A. Laboratoire de Génétique biochimique, Centre de Recherches Zootechniques \\ F 78350 Jouy-en-Josas
}

\begin{abstract}
Résumé
Le phénotype d'un veau Charolais au système de groupes sanguins S s'explique en supposant que l'animal a reçu un allèle recombiné, apparu soit chez l'un de ses parents, soit chez son grand-père paternel, le crossing over s'étant produit entre les déterminants génétiques des facteurs $\mathbf{U}, \mathrm{H}^{\prime \prime}-$ et sans doute $\mathrm{H}^{\prime}$ - et celui du facteur $\mathbf{U}^{\prime \prime}$. Ce cas probable de recombinaison est le premier signalé pour le système $S$. Il est discuté à la lumière des particularités de la série des phénogroupes répertoriés dans les races françaises.
\end{abstract}

Mots clés : Groupes sanguins, bovins, système $S$, recombinaison.

\section{Summary}

$A$ probable case of recombination within the $S$ system of cattle blood groups

An unusual $\mathbf{S}$ system phenotype noted in a Charolais calf may be accounted for by supposing that the animal inherited a recombinant allele, originating from either parent or from its parental grand sire, the crossing-over having taken place between the genetic determinants of factors $\mathbf{U}, \mathbf{H}^{\prime \prime}$ - and very likely $\mathrm{H}^{\prime}$. - and that of factor $\mathrm{U}^{\prime \prime}$. This probable case of recombination is the first to be reported for the S system. It is discussed in the context of the particular phenogroup series found in french breeds.

Key words : Blood groups, cattle, $S$ system, recombination.

\section{Introduction}

Sur les quelques 80 réactifs monospécifiques mis au point dans le monde pour l'analyse des groupes sanguins des bovins, 8 détectent des facteurs antigéniques appartenant au système $\mathrm{S}: \mathrm{S}, \mathrm{U}, \mathrm{H}^{\prime}, \mathrm{U}^{\prime}$ (STORMONT et al., 1961 ; nomenclature d'époque : $S_{1}$ pour $S$ et $U_{1}$ pour $U$ ), $U^{\prime \prime}, \mathrm{H}^{\prime \prime}$ (Grosclaude \& Millot, 1963 ; nomenclature d'époque : $U_{2}^{\beta}$ pour $\left.H^{\prime \prime}\right), S^{\prime \prime}$ (Grosclaude, 1963) et $U_{2}^{\prime}$ (Grosclaude, 1965). On peut 
par ailleurs obtenir plusieurs autres anticorps qui ont la propriété de détecter deux ou

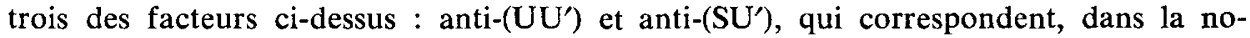


anti-(SU") (Grosclaude \& Millot, 1963) ; ces anticorps, appelés anticorps "secondaires: 》 par Grosclaude \& Millot (1963), par opposition aux anticorps «primaires 》 spécifiques des facteurs $\mathrm{S}, \mathrm{U}, \mathrm{H}^{\prime}, \mathrm{U}^{\prime}, \mathrm{U}_{2}^{\prime}, \mathrm{H}^{\prime \prime}, \mathrm{S}^{\prime \prime}$ et $\mathrm{U}^{\prime \prime}$, ne sont pas utiles à l'analyse du polymorphisme du système si l'on dispose des anticorps primaires.

A l'image de ce qui s'observe dans les systèmes $B$ et $C$, la plupart des allèles du système $S$ contrôlent plus d'un facteur antigénique. On utilise donc, ici aussi, le terme de «phénogroupe» pour désigner les groupes de facteurs contrôlés par ces allèles. Au total 17 phénogroupes ont été identifiés pour le moment dans les races françaises : $\mathrm{SH}^{\prime}, \mathrm{SH}^{\prime} \mathrm{S}^{\prime \prime}, \mathrm{SH}^{\prime} \mathrm{S}^{\prime \prime} \mathrm{U}^{\prime \prime}, \mathrm{SH}^{\prime} \mathrm{U}^{\prime \prime}, \mathrm{UH}^{\prime} \mathrm{H}^{\prime \prime}, \mathrm{UH}^{\prime} \mathrm{H}^{\prime \prime} \mathrm{U}^{\prime \prime}, \mathrm{H}^{\prime}, \mathrm{H}^{\prime} \mathrm{S}^{\prime \prime}, \mathrm{H}^{\prime} \mathrm{H}^{\prime \prime}, \mathrm{H}^{\prime} \mathrm{U}^{\prime \prime}, \mathrm{H}^{\prime} \mathrm{H}^{\prime \prime} \mathrm{U}^{\prime \prime}$, $U^{\prime} U_{2}^{\prime}, U^{\prime} U_{2}^{\prime} U^{\prime \prime}, U_{2}^{\prime}, U_{2}^{\prime} U^{\prime \prime}, U^{\prime \prime}$ et - (phénogroupe négatif) ; l'existence chez des bovins hongrois (ESABR, 1965) d'un dix-huitième phénogroupe (vraisemblablement $\mathrm{UH}^{\prime}$ ) a été vérifiée (Grosclaude, 1965), et plusieurs autres phénogroupes existent dans des races africaines (Grosclaude, résultats non publiés). Sur les 11 systèmes de groupes sanguins actuellement connus chez les bovins, le système $S$ se classe ainsi au troisième rang, après les systèmes $B$ et $C$, pour le degré de polymorphisme.

L'analyse des cas de recombinaison observés au sein des systèmes $\mathbf{B}$ et $\mathbf{C}$ a permis d'entreprendre l'établissement de la carte génétique de ces systèmes, les ébauches les plus récentes étant dues à Grosclaude et al. (1982) pour le système B, et Guerin et al. (1981) pour le système $\mathrm{C}$; elle a aussi permis de conclure que les phénogroupes de chacun de ces deux systèmes étaient contrôlés par un ensemble de gènes étroitement liés. Par contre, aucun cas de recombinaison n'avait encore été signalé dans le système S. La présente note rapporte un premier cas probable, qui permet d'aborder l'analyse de la structure génétique de ce système.

\section{Matériel et méthodes}

L'étude porte sur la filiation d'une génisse de race Charolaise, née le 3-4-1983. La compatibilité du groupe sanguin de ce veau avec ceux de ses parents a été vérifiée dans le cadre du programme de contrôles systématiques réalisé par le Herd-Book de la race (environ 5000 analyses par an). Les groupes sanguins des parents avaient déjà été établis, celui de la mère (née en 1971) en 1976, celui du père (né en 1981), en 1981. Le groupe sanguin du veau a été déterminé à partir d'un échantillon de sang prélevé vers l'âge de 5 mois ; un nouvel échantillon a été prélevé 1 mois plus tard, pour répéter l'analyse et procéder à des vérifications. Par ailleurs, les groupes sanguins de 4 produits de la mère avaient été établis au cours des années précédentes; par contre le veau est le seul descendant actuellement connu du père.

Les tests hémolytiques et les absorptions ont été effectués comme décrit dans Grosclaude et al. (1979). Les 8 réactifs du système $\mathrm{S}$ (anti-S, U, $\mathrm{H}^{\prime}, \mathrm{U}^{\prime}, \mathrm{U}_{2}^{\prime}, \mathrm{H}^{\prime \prime}$, $\mathrm{S}^{\prime \prime}$ et $\mathrm{U}^{\prime \prime}$ ) ont été régulièrement comparés à ceux des autres Laboratoires à l'occasion des Tests de Comparaison Internationaux organisés tous les deux ans par la Société Internationale pour l'Etude des Groupes sanguins des Animaux (I.S.A.B.R.). Ces réactifs sont tous parfaitement fiables. 


\section{Résultats}

La figure 1 récapitule les données acquises sur le phénotype du veau au système $S$, et sur le phénotype, ou le génotype lorsqu'il est connu, de ses parents et grandsparents. La présence des facteurs $S$ et $U^{\prime \prime}$ ainsi que l'absence du facteur $U$ chez le veau ont été vérifiées par absorption; en ce qui concerne $U^{\prime}$, l'utilisation parallèle d'anti- $U^{\prime}$ et anti- $U_{2}^{\prime}$ donne un recoupement. Les génotypes de la mère et du grandpère paternel ont pu être déduits sans ambiguïté de données sur leurs descendants. On constate que le veau a reçu de sa mère le facteur $S$, et sans doute le facteur $\mathbf{H}^{\prime}$, et de son père les facteurs $\mathrm{U}^{\prime}$ et $\mathrm{U}_{2}^{\prime}$ qui proviennent de son grand-père paternel. Par contre, la présence dans son groupe sanguin du facteur $U^{\prime \prime}$ est inattendue. Elle peut s'expliquer en supposant la survenue, dans la séquence d'ADN codant le phénogroupe $\mathrm{UH}^{\prime} \mathrm{H}^{\prime \prime} \mathrm{U}^{\prime \prime}$, d'un crossing over entre les déterminants des facteurs $\mathrm{U}$ et $\mathrm{H}^{\prime \prime}$ et celui du facteur $U^{\prime \prime}$. Les données actuelles ne permettent pas de dire si le gamète recombiné est apparu chez la mère, chez le père, ou chez le grand-père paternel. La figure 2 montre que, dans le premier cas, le phénogroupe recombiné s'écrirait SH'U", dans les deux autres cas, $U^{\prime} U_{2}^{\prime} U^{\prime \prime}\left(\mathrm{H}^{\prime} U^{\prime} U^{\prime}{ }_{2} U^{\prime \prime}\right.$ étant très peu probable compte tenu de l'étroite association entre $U$ et $\mathbf{H}^{\prime}$ ). L'analyse des groupes sanguins de futurs descendants du veau et de son père permettrait de préciser ce point.

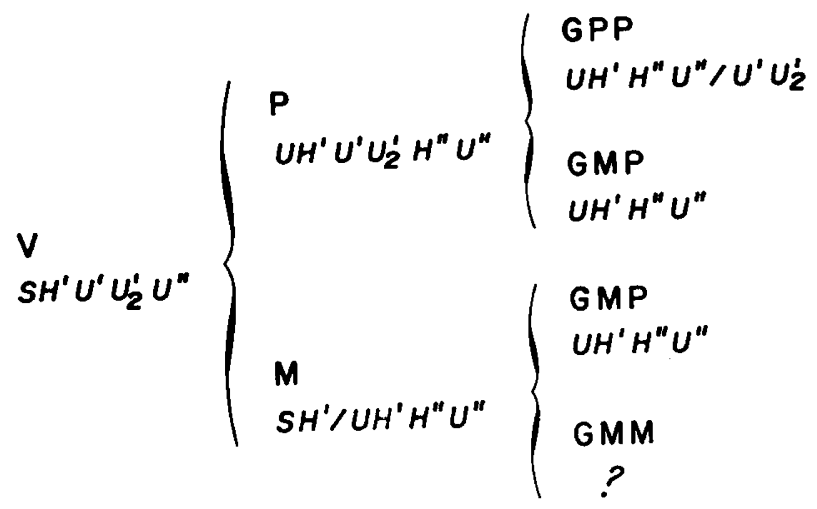

FIG. 1

Phénotype au système $S$, ou génotype lorsqu'il est connu avec certitude, du veau (V) et de ses ascendants ( $P$ : père; $M$ : mère; GPP : grand-père paternel, etc.). ?: groupe sanguin non déterminé.

$S$ system phenotype, or genotype when it is known with certainty, of the calf (V) and of its ascendants (P: sire; $M$ : dam; GPP : paternal grand-sire, etc.). $?:$ blood type not established. 


\section{Discussion}

L'apparition chez un produit d'un phénogroupe remanié par recombinaison fait paraître sa filiation incompatible. De manière générale, il se pose donc le problème de distinguer les filiations vraies avec recombinaison des filiations fausses, problème qui a été discuté précédemment (Grosclaude, 1982). Trois paramètres sont à prendre en compte : le degré de fiabilité des informations généalogiques disponibles, mesurable par le taux d'erreurs de filiation dans la population ou l'élevage considéré ; la fréquence dans cette population ou cet élevage du phénogroupe s'écrivant comme le phénogroupe recombiné, s'il existe; la probabilité de la recombinaison susceptible de produire ce phénogroupe recombiné.

Dans le cas présent, les informations généalogiques peuvent être considérées comme relativement fiables. En effet, depuis 1971, sur 107 filiations vérifiées dans l'élevage naisseur du veau, 3 seulement ont été trouvées incompatibles, la dernière en 1979 ; dans les 3 cas la filiation correcte a pu être retrouvée sur une nouvelle proposition de l'éleveur. Par ailleurs, aucun des deux phénogroupes inattendus qu'a pu recevoir le veau, $\mathrm{SH}^{\prime} \mathrm{U}^{\prime \prime}$ ou $\mathrm{U}^{\prime} \mathrm{U}_{2}^{\prime} \mathrm{U}^{\prime \prime}$, n'a été observé dans l'élevage. Le premier n'a d'ailleurs pas été trouvé jusqu'ici dans la race Charolaise. Le second y est très rare : il n'a été identifié que 4 fois parmi les quelques 7000 taureaux de la race dont le groupe sanguin est fiché ; même si ce phénogroupe existe à l'état masqué chez deux ou trois autres taureaux, sa fréquence est en définitive très faible, de l'ordre de $5 \cdot 10^{-4}$. L'ensemble de ces éléments conduit à admettre que le cas étudié est bien celui d'une filiation exacte avec recombinaison.

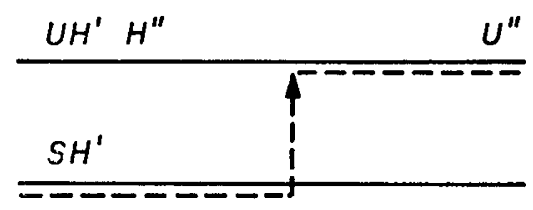

(M)

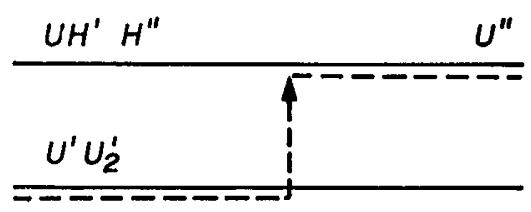

\section{( $P$ ou GPP)}

FIG. 2

Les deux types de crossing over supposés chez un ascendant de $V$ : production, chez $M$, d'un gamète ayant transmis le phénogroupe $S H^{\prime} U^{\prime \prime}$, ou production, chez $P$ ou GPP, d'un gamète ayant transmis le phénogroupe $U^{\prime} U_{2}^{\prime} U^{\prime \prime}$.

The alternative crossovers postulated in an ascendant of $V:$ in $M$, production of a gamete having transmitted phenogroup $S^{\prime} U^{\prime \prime}$; in $P$ or GPP, production of a gamete having transmitted phenogroup $U^{\prime} U_{2}^{\prime} U^{\prime \prime}$. 
Le fait que cette recombinaison se soit produite entre les déterminants des facteurs $U$ et $\mathbf{H}^{\prime \prime}$ et celui du facteur $U^{\prime \prime}$ peut être rapproché des particularités de la série de phénogroupes identifiés dans les races françaises et rappelée dans l'Introduction. Dans cette série, tout se passe comme si les déterminants génétiques des facteurs $S$, $U$ et $U^{\prime}$ étaient allèles, puisqu'on n'observe jamais plus d'un de ces facteurs dans un phénogroupe. Par ailleurs il existe une certaine homologie entre trois couples de facteurs : $\mathrm{S}$ et $\mathrm{S}^{\prime \prime}$, $\mathbf{U}$ et $\mathbf{H}^{\prime \prime}, \mathrm{U}^{\prime}$ et $\mathbf{U}_{2}^{\prime}$, les facteurs de chaque couple étant préférentiellement associés. Si l'association paraît plus étroite pour les deux derniers couples, on trouve toutefois couramment, chez les zébus africains (Grosclaude, résultats non publiés), $U$ sans $H^{\prime \prime}$ et $U^{\prime}$ sans $U_{22}^{\prime}$. Les déterminants génétiques des facteurs $S^{\prime \prime}$, $\mathrm{H}^{\prime \prime}$ et $\mathrm{U}_{2}^{\prime}$ sont donc bien distincts de ceux — peut-être allèles — de $S$, $U$ et $U^{\prime}$, mais paraissent leur être très étroitement liés. Par contre, le facteur $U^{\prime \prime}$, qui fait partie de 8 des 17 phénogroupes répertoriés en France, est indifféremment associé ou non à chacun des autres facteurs, y compris $\mathrm{H}^{\prime}$ (pour ce dernier facteur, on comparera au fait que $\mathrm{S}, \mathrm{U}, \mathrm{H}^{\prime \prime}$ et $\mathrm{S}^{\prime \prime}$ s'observent toujours avec $\mathrm{H}^{\prime}$ et que $\mathrm{U}^{\prime}$ et $\mathrm{U}_{2}^{\prime}$ s'observent toujours sans $\mathrm{H}^{\prime}$ ). On peut donc supposer que le déterminant génétique de $\mathrm{U}^{\prime \prime}$ est, toutes proportions gardées, relativement distant des autres déterminants du système. $\mathrm{Si}$ on accepte cette analyse, la recombinaison entre les déterminants de $\mathrm{U}$ et $\mathbf{H}^{\prime \prime}$ et celui de $\mathrm{U}^{\prime \prime}$, dont il a été fait état dans la présente note, appartient à la classe des recombinaisons les plus probables dans le système.

Reçu le 18 octobre 1983.

Accepté le 21 novembre 1983.

\section{Références bibliographiques}

E.S.A.B.R., 1965. Reference and Comparison Tests (Blood typing of cattle), Wageningen, The Netherlands. Document ronéotypé.

Grosclaude F., 1963. Allèles du locus $\mathrm{S}$ de groupes sanguins des bovins. Nouveaux résultats. Ann. Biol. Anim. Bioch. Biophys., 3, 433-435.

Grosclaude F., 1965. Studies on the $S$ blood group system in french cattle breeds. 9th European Conference on animal blood groups, Prague, 18-22 August 1964, Matoušek J. (ed.), Blood groups of animals, 79-85, Publishing House of the Czechoslovak Academy of Sciences, Prague.

Grosclaude F., 1982. Genetic recombination within cattle blood group systems and parentage control. 2nd World Congress of Genetics applied to Livestock Production, Madrid, 4-8 October 1982, 6, 350-358, Garsi, Madrid.

Grosclaude F., Guerin G., Houlier G., 1979. The genetic map of the B system of cattle blood groups as observed in French breeds. Anim. Blood Grps Biochem. Genet., 10, 199-218.

Grosclaude F., Lefebvre J., Noe G., 1982. Nouvelles précisions sur la carte génétique du système de groupes sanguins B des bovins. Génét. Sél. Evol., 15, 45-54.

Grosclaude F., Millot P., 1963. Allèles supplémentaires au locus S de groupes sanguins des bovins. Ann. Biol. Anim. Bioch. Biophys., 3, 119-124.

Guerin G., Grosclaude F., Houlier G., 1981. The C system of cattle blood groups. 2. - Partial genetic map of the system. Anim. Blood Grps Biochem. Genet., 12, 15-21.

Stormont C., Miller W.J., SuzuKi Y., 1961. The S system of bovine blood groups. Genetics, 46, 541-551. 